\title{
One Health: perspectives on ethical issues and evidence from animal experiments
}

\author{
G.V. Asokan, ' Z. Fedorowicz, ${ }^{2}$ P. Tharyan ${ }^{3}$ and A. Vanitha ${ }^{4}$
}

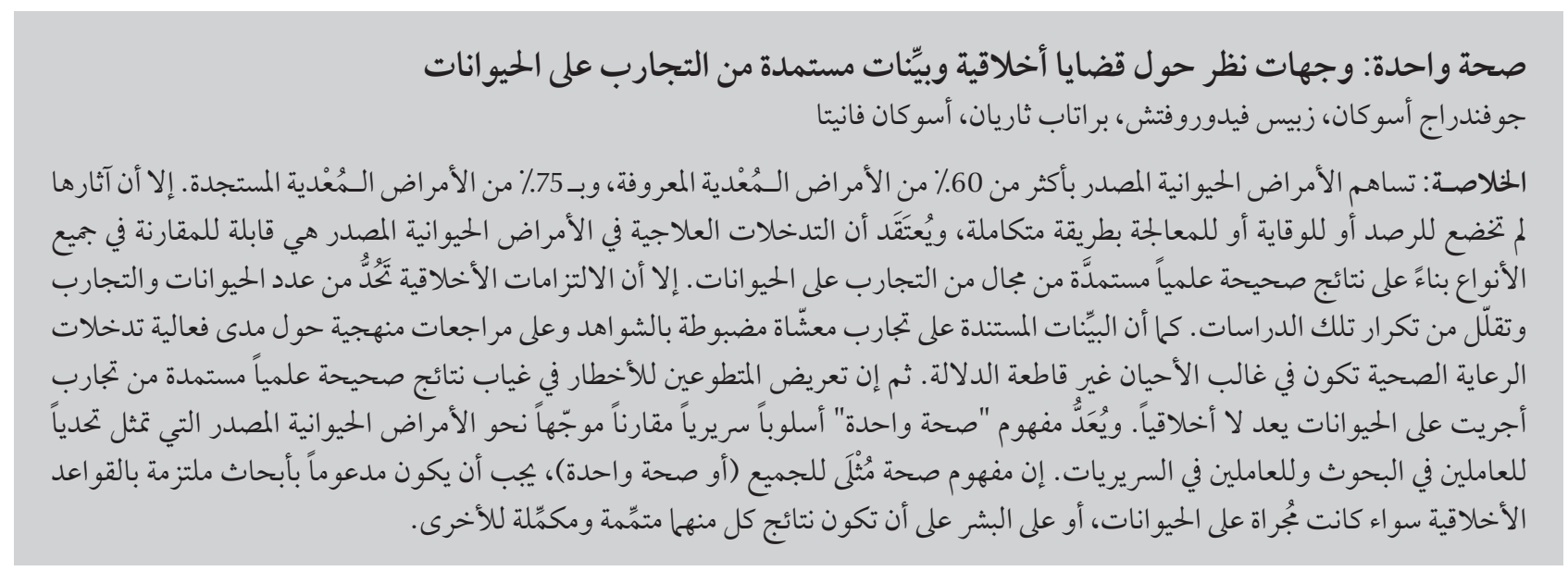

ABSTRACT Zoonoses constitute more than $60 \%$ of all known infectious diseases and $75 \%$ of emerging infectious diseases. Their impact is not monitored, prevented and treated in an integrated way. The efficacy of therapeutic interventions for zoonotic diseases is deemed to be comparable across species with scientifically valid results originating from a range of animal experiments. Ethical obligations limit the number of animals used in experiments as well as reduce repetition of studies. The evidence based on randomized controlled trails and systematic reviews for the effectiveness of health care interventions is often inconclusive. Subjecting human volunteers to risk in the absence of scientifically valid results from animal experiments is unethical. The One Health concept is a comparative, clinical approach directed towards zoonoses which present challenges to research workers and clinicians. Optimal health for all-One Health-should be underpinned by ethically conducted research in animals or humans and the results should be complementary to both.

One health : perspectives sur les questions éthiques et les preuves obtenues à partir d'expérimentations sur les animaux

RÉSUMÉ Les zoonoses représentent plus de $60 \%$ de toutes les maladies infectieuses connues et $75 \%$ des maladies infectieuses émergentes. Leur impact ne fait pas l'objet d'une surveillance et il n'est ni prévenu, ni traité de manière intégrée. L'efficacité des interventions thérapeutiques pour les zoonoses est jugée comparable entre les espèces avec des résultats scientifiquement valides issus d'un éventail d'expérimentations sur les animaux. Les obligations éthiques limitent le nombre d'animaux utilisés dans les expériences et réduisent la répétition des études. Les preuves issues d'essais randomisés et contrôlés et les revues systématiques de l'efficacité des interventions des soins de santé sont souvent non concluantes. Exposer des volontaires humains au risque en absence de résultats scientifiquement validés issus d'expérimentations sur les animaux est contraire à l'éthique. Le concept One health (une seule santé) est une approche clinique comparative visant les zoonoses qui représentent des défis pour les chercheurs et les cliniciens. Le concept d'une santé optimale pour tous, One health, doit être étayé par une recherche respectant l'éthique pour les animaux ou les humains et les résultats doivent être complémentaires pour les deux.

'Public Health Programme, College of Health Sciences, University of Bahrain; ${ }^{2}$ Bahrain Branch of UK Cochrane Centre, Ministry of Health, Manama, Bahrain (Correspondence to G.V.Asokan:agv@health.gov.bh).

${ }^{3}$ South Asian Cochrane Centre and Network, Christian Medical College, Vellore, India.

${ }^{4}$ American Mission Hospital, Manama, Bahrain.

Received: 25/08/11; accepted: 11/12/11 


\section{Introduction}

One Health is a concept based on a systems approach which amalgamates the "collaborative effort of multiple disciplines working locally, nationally, and globally to attain optimal health for people, animals and our environment" [1]. It can be seen as a strategy for expanding interdisciplinary collaboration and communication in all aspects of health care for humans and animals and more especially within the context of zoonoses. The World Health Organization estimates that $25 \%$ of 57 million deaths per annum that occur globally are caused by microbes [2]. A comprehensive literature review by Taylor et al. identified 1415 species of infectious human pathogens [3]. Zoonotic diseases constitute more than $60 \%$ of all known infectious diseases, with humans serving as the primary reservoir for only $3 \%$ of them, and of the 175 species of infectious organisms considered to be emerging a large percentage (75\%) are zoonotic.

The impact of zoonotic diseases on human and animal health is not monitored, prevented and treated in an integrated way, despite the fact that the etiologies and treatments of these diseases are generally similar across species. The efficacy of therapeutic interventions in zoonoses is also believed to be similar across species and it is prudent to demand scientifically valid evidence of efficacy - an obligation in animal experiments for newer drugsthat are applicable to multiple species including humans. There is an increased tendency, coupled with more stringent ethical obligations, towards limiting the number of animals used in experiments while at the same time ensuring that the replication of previous research is reduced.

This paper is a brief discussion of evidence and ethics in animal experiments from a One Health perspective, against a backdrop of an expanding number and species of zoonoses.

\section{A "numbers game" in animal experiments}

Around 50 to 100 million vertebrate animals are used worldwide annually for research and experiments. These continue to further the development of our understanding of the functioning of both the human and animal body [4]. The 3 Rs of humane animal experimentation-Replacement, Reduction and Refinement-are widely considered to be the guiding principles for the use of animals in research, where Reduction refers to methods that enable researchers to obtain comparable levels of information from fewer animals or to obtain more information from the same numbers of animals [5].

The 3 concerns addressed in this commentary are the reduction in animal experimentation in the domain of zoonoses; interdisciplinary One Health; and ethical considerations for humans and other species.

\section{Does the principle of reduction compromise the sample size required for scientific validity under the pretext of ethics?}

Choosing the sample size in a scientific experiment involves balancing the increased information and precision that results from bigger samples against the reduced time and cost that arise from smaller samples. In practice, scientists undertaking animal research justify the number of animals to be used, and committees supervising animal experimentation review this justification for approval.

Using group sizes of 6 or 8 animals regardless of the type of experiment or number of groups is customary in some disciplines, which may be inappropriate in factorial experimental designs or designs with more than 2-3 treatment groups. However, although the conventional sample size of 6 or 8 may be considered appropriate in pilot or exploratory studies [6], this is still open to debate. Notably in animal experiments where welfare remains an issue, there is an additional motive to reduce the numbers of animals that undergo experiments or are sacrificed.

\section{Can the evidence from such a reduction in animal experiments be carried forward to human trials?}

Translating and extrapolating the results of animal experiments to provide reliable evidence of the potential benefits to humans faces similar constraints. Pablo et al. for example conducted a systematic review of 6 interventions providing evidence of a treatment effect, either benefit or harm, in clinical trials [7]. They looked for concordance with the corresponding animal experiments, and concluded that agreement between animal studies and clinical studies varied, in that 3 studies had similar outcomes and 3 did not. Furthermore they concluded that this lack of concordance between animal experiments and clinical trials may be due to bias, random error or the failure of animal models to adequately represent human disease.

Recent results from metabolomics and proteomics studies revealed that the effect size observed in human studies was strikingly lower when compared with matching animal studies [8]. Similarly, in a review on effectiveness of therapies in the treatment of peri-implantitis, the characteristics of the studies were extremely heterogeneous as no animal experiment and human trial had comparable study procedures, including sample size and power [9]. A Cochrane systematic review on antiretroviral post exposure prophylaxis (PEP) for occupational HIV exposure concluded that current clinical practice is based on results from individual primary animal studies, and recommended a formal systematic review of all relevant animal studies [10]. Overall the limitations in translating the results of animal 
experiments include, among others, the non-availability of suitable animal models, clinical heterogeneity and predominantly inadequate sample sizes leading to effect size bias.

\section{Is it ethical to carry out human trials based on the uncertain or inconclusive results of animal experiments?}

Even after the inclusion of a substantial number of randomized controlled trials, many systematic reviews, for example those published in the Cochrane library, are still unable to provide conclusive clinical recommendations. Clearly it is the biased or imprecise results from animal experiments that result in clinical trials of biologically inert or even harmful substances, thus exposing patients to unnecessary risk. Besides, scarce research resources such as grants for research, scientific human resources and materials, including experimental animals, are being wasted. Moreover, animals suffer unnecessarily if animal experiments fail to inform medical research adequately.

\section{Whose ethics are we concerned most with or should there be equity across species in addressing the ethical issues arising in clinical experiments?}

It is unethical to subject healthy human volunteers to risk in the absence of precise and scientifically valid results from animal experiments? Clearly the anthropocentric attitude of investigators, who may not have a clear understanding of the dynamics of the disease across species, can result in healthy human volunteers being subjected to unnecessary risk. While designing animal experiments the consideration of ethics should not just be limited to the animals alone but also to multiple species to whom the results are then taken forward. In the case of zoonoses such inadequacies in evidence from animal experiments are shared risks which affect not only humans but also multiple species.

\section{Conclusion}

Broadly speaking, animal experiments play an important part in the chain of research evidence and as such are used to decide which interventions are taken forward in clinical trials. Efforts to minimize bias and random error are therefore as important when reviewing the results of animal models as when reviewing the results of clinical trials in humans. Conversely, the repetition of experiments is considered necessary for the improvement of the precision and reliability of the results [11]. An increase in the number of animals included in experiments will improve the precision and reliability of the results and ultimately their generalizability.

The One Health concept is a comparative clinical approach which takes into consideration the "shared risks" between humans and animals concerning zoonoses and in this way it promotes better cooperation and collaboration between human and animal health professionals to identify and reduce such risks. To determine similarities between animal models and clinical trials, researchers engaged in animal experiments need to direct their attention to the following:

- Standardization of research procedures to reduce heterogeneity between animal and human studies.

- Power analyses and sample-size calculation in animal experiments.

- Prospective registration of animal experiments, similar to registration of clinical trials on humans. This will go a long way towards reducing publication bias.

- Producing evidence through systematic reviews and meta-analyses of animal experiments for comparison with clinical trials.

A recent commentary published in the Journal of Evidence-based Medicine proposed that the Cochrane Collaboration should consider registering a new group dedicated to conducting systematic reviews on zoonotic diseases thus underpinning the systems approach and One Health [12].

In summary, researchers should not only avoid using more animals for experiments than needed, they should also aim to avoid using too few. Either way can result in unethical research that is wasteful of resources and time and that produces results that are of limited relevance, most notably for zoonoses.

\section{References}

1. One Health. American Veterinary Medical Association [website] (https://www.avma.org/KB/Resources/Reference/Pages/One-Health94.aspx, accessed 23 September 2012).

2. Chugh TD. Emerging and re-emerging bacterial diseases in India. Journal of Biosciences, 2008, 33:549-555.

3. Taylor LH, Latham SM, Woolhouse MEJ. Risk factors for human disease emergence. Philosophical Transactions of the Royal Society of London. Series B, Biological Sciences, 2001, 356:983-989.

4. Barbara Orlans F. History and ethical regulation of animal experimentation: an international perspective. In: Kuhse $\mathrm{H}$,
Singer P, eds. A companion to bioethics, 2nd ed. New Jersey, Wiley-Blackwell, 2009:399-409.

5. Flecknell P. Replacement, reduction and refinement. ALTEX, 2002, 19:73-78.

6. Sample size. Isogenic.Info [website] (http://www.isogenic.info/ html/13_sample_size.html, accessed 18 September 2012).

7. Perel $\mathrm{P}$ et al. Comparison of treatment effects between animal experiments and clinical trials: systematic review. British Medical Journal, 2007, 334:197.

8. Guo Yu et al. Sample size and statistical power considerations in high-dimensionality data settings: a comparative study of classification algorithms. BMC Bioinformatics, 2010, 11:447. 
9. Faggion CM Jr, Schmitter M, Tu YK. Assessment of replicał tion of research evidence from animals to humans in studies on peri-implantitis therapy. Journal of Dentistry, 2009, 37:737-747.

10. Young TN et al. Antiretroviral post-exposure prophylaxis (PEP) for occupational HIV exposure. Cochrane Database of Systematic Reviews, 2007, (1):CD002835.

11. Schneider B. Begründung der Wiederholung von Tierversuchen und Bestimmung der erforderlichen Tierzahl gemäss dem
Deutschen Tierschutzgesetz. [Justification of repeated animal experiments and determination of the required number of animals according to the German Animal Protection Act]. Arzneimittel-Forschung, 2009, 59:318-325.

12. Asokan G et al. Use of a systems approach and evidence-based One Health for zoonoses research. Journal of Evidence-Based Medicine, 2011, 4:62-65. 